\title{
Brain Tissue Classification to Detect Focal Cortical Dysplasia in Magnetic Resonance Imaging
}

\author{
Fabrício H. Simozo ${ }^{1}$, Marcos S. Oliveira², Luiz O. Murta-Junior ${ }^{2}$ \\ ${ }^{1}$ Departamento de Física - FFCLRP - Universidade de São Paulo (USP). \\ ${ }^{21}$ Departamento de Computação e Matemática - FFCLRP - Universidade de São Paulo \\ (USP). \\ Av. Bandeirantes, 3900 - Monte Alegre -Ribeirão Preto - SP \\ \{fsimozo, marcossoares10, murta\} @usp.br
}

\begin{abstract}
Focal cortical dysplasia (FCD) is a local malformation of the cortex, the main cause of refractory epilepsy in childhood and one of the most common causes in adults. The surgery decision and planning depend on the FCD localization. Although recent studies have successfully detected FCD through artificial intelligence, no study investigates the relevance and prevalence of cortical features on FCD identification and the performance of different machine learning techniques. In this study, the proposed method constructed a voxel-based set of features, e.g., texture measure, border definition, cortical thickness.
\end{abstract}

Resumo. A displasia cortical focal (CDF) é uma malformação local do córtex, a principal causa de epilepsia refratária na infância e uma das causas mais comuns em adultos. A decisão e o planejamento da cirurgia dependem da localização do FCD. Embora estudos recentes tenham detectado com sucesso o FCD por meio da inteligência artificial, nenhum estudo investiga a relevância e a prevalência de características corticais na identificação do FCD e no desempenho de diferentes técnicas de aprendizado de máquina. Neste estudo, o método proposto construiu um conjunto de recursos baseados em voxel, por exemplo, medida de textura, definição de borda, espessura cortical.

\section{Introduction}

Focal cortical dysplasia (FCD) is the prevalent cause of refractory epilepsy in children and one of the primary causes of such epilepsy in adults (Krsek et al., 2008). Refractory epilepsy is a condition in which the patients are irresponsive to antiepileptic drugs, affecting approximately $30 \%$ of epilepsy patients. The reoccurrence of seizures may cause irreversible damage to brain development and quality of life (Roy et al., 2011; Sheppard \& Lippé, 2012).

In the case of FCD epilepsy, the removal or disconnection of the lesion region, which originates the abnormal electrical discharges, may prevent or lower the occurrence of new epileptic seizures. For that reason, identifying lesion location and its extension is essential to guarantee a favorable surgery outcome. 
However, this task is often not easy. FCD defines a highly heterogeneous group of lesions, with different clinical, histological and medical images characterization, and even different surgical responses (Colombo et al., 2003; Fauser et al., 2004; Francione et al., 2003; Hildebrandt et al., 2005; Krsek et al., 2008).

The International League Against Epilepsy (ILAE) has a definition of classifications on the various types of FCD (Blümcke, Thom, \& Aronica, 2011) and guidelines for clinical (Fisher et al., 2014), surgical (Blümcke et al., 2016; Fisher et al., 2017). However, there are not many quantitative image characterization studies and reports.

FCD localization may be a hard task due to the highly heterogeneous feature, often demanding the use of numerous techniques and protocols, i.e., structural and functional tomography, scalp, and depth electroencephalogram. In recent years, MRI analyses have become an essential factor for clinical decisions (Ramli, Rahmat, Lim, \& Tan, 2015). The use of automatic methods to assist identification of FCDs show promising results (Kini, Gee, \& Litt, 2016), but is not yet widespread due to nonintuitive interfaces and lack of availability of proposed methods (Duncan et al., 2016).

Several approaches are possible when using automated computational algorithms to assist FCD segmentation (Adler et al., 2017; Ahmed et al., 2015; Bergo \& Falcao, 2008; Cantor-Rivera, Khan, Goubran, Mirsattari, \& Peters, 2015; Hong et al., 2014) or to create maps to enhance the contrast of FCD lesions for manual segmentation (Antel et al., 2003; Bernasconi et al., 2001; Huppertz, 2013). In most cases, results from these studies are reported without specific quantitative details, open Datasets, or even open-source codes provided, handicapping further development and clinical testing of proposed methods.

It is necessary sharing source codes for FCD detection methods over the scientific communities to boost the use of automatic methods for assisting treatment in epilepsy patients with FCD, and for those methods to be developed and distributed in ways that facilitate use by different institutions and professionals (Duncan et al., 2016). Furthermore, the creation of a Database with computer extracted features and terminology that is accessible to clinicians and specialists is a big step towards the use of said methods in clinical environments and has supported advancements in identification for other brain lesions such as Multiple Sclerosis and brain tumors (Carass et al., 2017; Hatt et al., 2018; Menze et al., 2015).

In this study, we propose a way of characterizing FCD lesions by using automatic preprocessing and feature extraction algorithms and classifying these features using machine learning algorithms. The efforts resulted in the creation of a public Database and an open-source segmentation algorithm in a 3DSlicer extension. This paper is organized as follows: material and methods describing database from patients and the methods of both Radiomics features and selected artificial intelligence employed, results show findings and discuss them in FCD detection, and finally, conclusions comments implications of this study and its limitations.

\section{Materials and Methods}

\subsection{Patient group}


We selected 15 patients treated at the Center of Surgery of Epilepsy (CIREP - Centro de Cirurgia de Epilepsia) of the Clinical Hospital of the Faculty of Medicine of Ribeirão Preto (HCFMRP - Hospital das Clínicas da Faculdade de Medicina de Ribeirão Preto), in Ribeirão Preto, São Paulo, Brazil, were selected for a study in FCD identification. These patients contained both volumetric T1 images and axial FLAIR images acquired in the same MRI exam. All patients were MRI positive for FCD type II ( $\mathrm{a}$ or $\mathrm{b}$ ), and the histological findings confirmed the image report.

\subsection{Control group}

Two groups containing images of asymptomatic subjects were selected to compose the control group, one with 30 FLAIR images and one with 40 3DT1 images. We used a Philips Achieva 3T Extra Series to acquire both patient and control group images in the HCFMRP CCIFM department.

\subsection{Image preprocessing}

All selected images underwent the same preprocessing pipeline. The pipeline involved N4 inhomogeneity filtering (ANTs) and skull stripping (FSL), resulting in an image containing only brain tissue and Cerebrospinal Fluid (CSF). Then, we normalized histogram using one of the control group images (the one with better visual White Matter (WM) x Grey Matter (GM) contrast) as a reference using the ImageMath algorithm from ANTs. We used the anisotropic diffusion filter (PeronaMalik) in all images to enhance Signal Noise Ratio.

For the patient group, T1 images were registered to the FLAIR images using a rigid + affine registration approach (ANTs). For the control groups, all images were registered to the MNI152 space, using a rigid + affine + SyN (ANTs) registration approach.

\subsection{Image processing}

For both groups, we used T1 images in three-class tissue segmentation using ATROPOS (ANTs) and cortical thickness estimate using DiReCT (ANTs), while we used FLAIR images for texture feature extraction.

We created and used Brain Tissue Characterization for feature extraction and mapping, i.e., a 3DSlicer module developed as a command-line interface (CLI) in C++ and available at Slicer Extension Manager. This algorithm calculates Haralick features for a moving window, resulting in multiple maps in which each voxel contains the value of each feature for the neighborhood $3 \times 3$ of that position on the original image. We calculated co-occurrence matrixes using eight intensity bins, four directions, and only for voxels inside the $3 \times 3$ neighborhood. The algorithm averages feature values for each direction for the final texture mapping result. We used a two-dimension neighborhood because input images were acquired in Axial FLAIR protocols, resulting in images with poor Z-axis resolution.

Extracted features are Contrast (Inertia), Cluster Prominence, Cluster Shade, Correlation, Energy, Entropy, Haralick Correlation, and Homogeneity (Inverse Different Moment). Together with image Intensity and Cortical Thickness, we have ten different features characterizing the cortex of each subject. These features are all extracted from original images after $\mathrm{N} 4$ and skull stripping corrections, and before registration avoiding transformation induced distortion is affecting generated maps 
exemplified in Figure 1. It is worth noting that the texture mappings were extracted from the whole brain and not only GM areas.

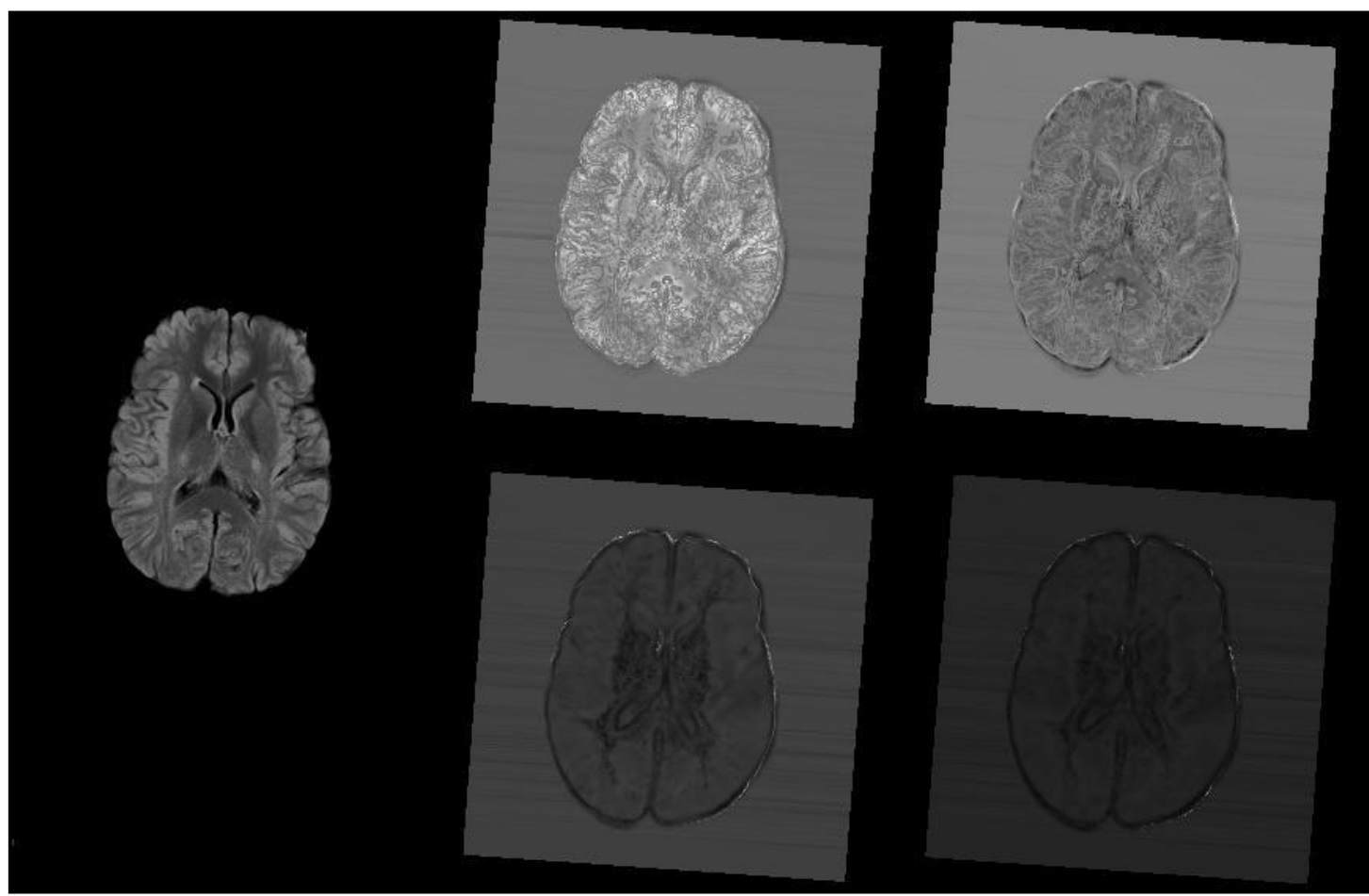

Figure 1: Example of texture feature maps extracted from one of the patients. One can see at left: Preprocessed image. Right, from top left to bottom right: Energy, Entropy, Haralick Correlation, and Contrast feature maps. Since features were calculated voxel-wise by considering the voxel neighborhood, it is possible to obtain a map with the same resolution of the original image.

\subsection{Manual FCD segmentation}

For images in the patient group, an expert neurologist manually segmented the FCD lesion. This segmentation was performed on FLAIR images using the 3DSlicer label editor. For two patients, some abnormalities did not configure an FCD. We also segmented these abnormalities with a different label identification. Therefore, resulting masks contained an FCD lesion for each patient, with eventual non-FCD lesion locations also indicated.

\subsection{Healthy Brain Templates}

By using control group images and the registration transform of each subject to the MNI152 space, we were able to create a template containing mean and standard deviation for each of the extracted features. With these templates, we can determine what values of each feature is expected in each region for a healthy brain, as each voxel has descriptive information regarding the value distribution in that region for all control group images.

\subsection{Distribution characterization and comparison}

With the defined H-FCD-masks, it is possible to characterize and classify the distribution of each feature inside the labels for each patient. For characterization, we 
present in three ways: Descriptive statistics of distributions, Statistical analysis with Kruskal-Wallis test, and dimensionality analysis.

\subsection{Identification - Data Mining classification}

We used a Random Forest classifier for FCD classification. We tested the classifier through a Leave-one-patient-out (LOPO) cross-validation, in which one train the classifier in all patients but one, and then tested on the data of that patient alone. This type of cross-validation ensures there is no patient-specific bias influencing the results.

Results obtained the form of a voxel-wise confusion matrix for the classification step, with the respective metrics. The confusion matrix indicates the distribution of sample classification regarding the real class of the sample and classification output of the sample. Hence, it indicates True Positives (TP), False Positives (FP), True Positives (TP), and True Negatives (TN). This data represents, quantitatively, how well the classifier performed in each training-testing set.

The metrics that can be extracted from these indicators are:

$$
\begin{aligned}
& \text { Precision }=\frac{T P}{T P+F P} \\
& \text { Recall }=\text { Sensitivity }=\frac{T P}{T P+F N} \\
& \text { Specificity }=\frac{T N}{T N+F P}
\end{aligned}
$$

From this step, we obtain the Classification Map. This map consists of a label mask in FLAIR image native space for the patient, indicating which samples, or voxels, were classified as healthy and which ones were classified as FCD. This data represents, qualitatively, which location of the cortex was identified by the classifier as FCD, perfectly fitting into the original image, allowing spatial analysis.

\subsection{Tissue classification}

Since the classification step is performed voxel-wise, the resulting Classification Map can have isolated FCD classifications outside of the FCD region (FP), as well as $\mathrm{H}$ classifications inside the FCD region (FN). A post-processing pipeline was developed to try and adjust a better way of representing the data visually to account for that.

For this purpose, a morphologic filter consisting of an opening and a dilation operation is used over the FCD classification. With this filter, all isolated FCDclassified voxels are eliminated, while the clusters of FCD-classified voxels are kept intact in the first step and then highlighted in the second step.

The downside of this step is that the quantitative spatial information of the classification loses its robustness, as the dilation step kernel size is arbitrarily chosen, and cannot be defined to have an adaptive size to avoid overgrowing the region. With this in mind, we can only say that the result of this final post-processing step is a qualitative indicator of the location of FCD classified clusters.

After obtaining the resulting FCD Map, we can extract the final metrics, which are measured in the larger scale of the presence or not of FCD volume in the region defined by the map. In this metric calculation, we defined the metrics as follows: 
-If the region indicated by the map is inside the manually segmented FCD region $(50 \%$ superposition), it configures a TP.

-If the region indicated by the map is not inside the manually segmented FCD region (50\% superposition), it configures an FP.

-If the manually segmented FCD does not have any region indicated by the map inside it (50\% superposition), it configures an FN.

-If there are more than one isolated region indicated by the map inside the same isolated manually segmented FCD, it will count as only one TP.

These metrics are presented as the results related to the capability of the proposed methodology in detecting FCD tissue automatically, as illustrated in Figure 2.

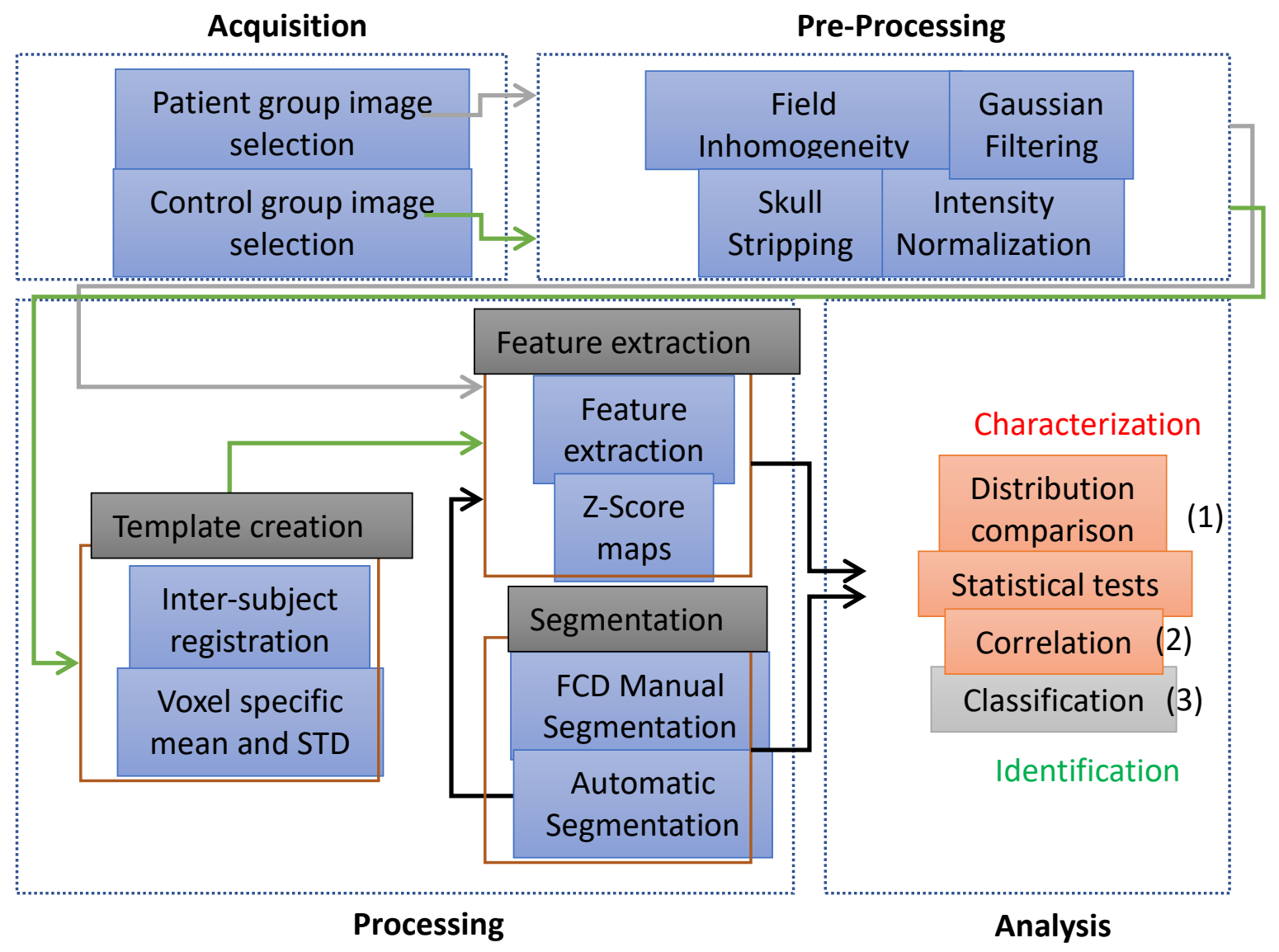

Figure 2. Full study pipeline. We performed the image acquisition step retrospectively using an image from the DICOM servers from HCFMRP. Preprocessing steps were performed using ANTs and FSL packages. Feature extraction was performed in both patient and control groups, and control groups feature images were used to create a template in MNI152 space. Automatic tissue segmentation and automatic segmentation were used to determine Healthy and FCD Cortex distributions of each feature. These distributions were used in the analysis steps to produce many quantitative values to characterize and identify FCD tissue. 


\section{Results and Discussion}

\subsection{Classification - voxel-wise analysis}

The results for this section are presented in the form of a confusion matrix for all classifications and classification metrics from each patient.

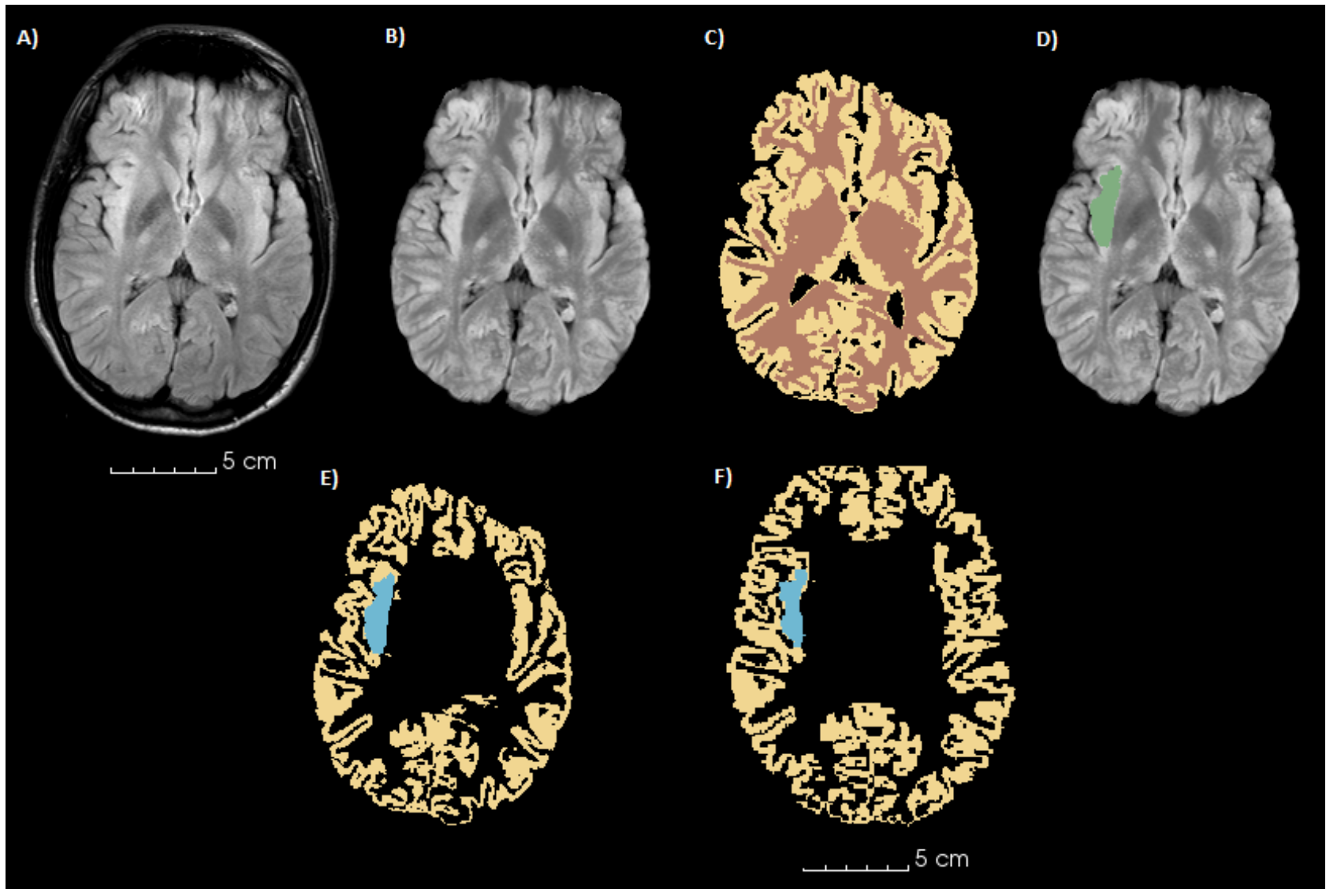

Figure 3: Illustration of preprocessing and segmentation pipeline steps. A) Original FLAIR image of a patient. B) Same FLAIR image after BET, Histogram normalization, and Perona Malik filtering. C) Automatic tissue segmentation obtained from the 3DT1 image registered to FLAIR space. D) Manual segmentation of FCD lesion made by a specialist. E) Analysis mask containing both automatic GM segmentation and manual FCD segmentation for the same patient. F) Analysis mask for the same patient registered to MNI152 space.

We proceeded the voxel to voxel classification, taking into account the features extracted from the neighborhood of each voxel that are passed to the classifier. Figure 3 illustrates a typical result of such classification showing the FCD voxels found by the classifier in green or light blue.

Table 1. Confusion Matrix for voxel-wise classification of Healthy Cortex and FCD tissue. This table includes all samples from each of the classifications performed for the LOPO cross-validation. These results show that $91 \%$ of the samples classified as DCF were correct, while only $16 \%$ of the DCF samples were classified as so. Because of the low coverage of the voxel-wise classification, the post-processing step was needed. 


\begin{tabular}{|l|l|l|l|l|l|l|}
\cline { 2 - 7 } \multicolumn{2}{c|}{} & \multicolumn{2}{l|}{ Classifier } & \multicolumn{2}{l|}{} \\
\cline { 2 - 7 } \multicolumn{2}{c|}{} & HC & FCD & Precision & Recall & Samples \\
\hline \multirow{3}{*}{ Real } & HC & 9370256 & 3787 & 0.98 & 1.00 & 9374043 \\
\cline { 2 - 7 } & FCD & 207447 & 40206 & 0.91 & 0.16 & 247653 \\
\hline
\end{tabular}

Table 2. Individual metrics for voxel-wise classification of Healthy Cortex and FCD tissue. This table includes the Precision and Recall metrics for the classification of each patient. It is clear that the precision score for most patients is high ( $>0.9$ on 10 out of 15 patients, $>0.6$ on 12 out of 15 patients), but the recall score is usually low (<0.2 on 9 out of 15 patients, $<0.4$ on 14 out of 15 patients).

\begin{tabular}{|l|l|l|}
\hline Patient & Precision & Recall \\
\hline 100 & 0,39 & 0,12 \\
\hline 101 & 0,99 & 0,11 \\
\hline 104 & 0,97 & 0,05 \\
\hline 107 & 0,92 & 0,2 \\
\hline 108 & 1 & 0,16 \\
\hline 111 & 0,98 & 0,12 \\
\hline 127 & 0,46 & 0,02 \\
\hline 128 & 0,81 & 0,26 \\
\hline 201 & 0,96 & 0,24 \\
\hline 202 & 0,68 & 0 \\
\hline 203 & 0,37 & 0,08 \\
\hline 205 & 0,99 & 0,6 \\
\hline 206 & 0,99 & 0,33 \\
\hline 207 & 0,96 & 0,14 \\
\hline 208 & 0,93 & 0,57 \\
\hline
\end{tabular}

Tables 1 and 2 evidence that the precision of the constructed automatic classifier is high (average of 0.91 for FCD classification), but the recall is low (average of 0.16 for FCD classification). The high specificity and low sensitivity indicate that, by using this method, the coverage of the resulting FCD lesion mask when compared with the ground-truth manual segmentation is low. On the other hand, if a region is classified as FCD in the resulting lesion mask, there is a high chance of it being a real FCD region.

When compared with recent results, this voxel-wise analysis is by the results obtained. Ahmed and collaborators proposed a Machine Learning classifier for T1 images by using morphological features and GM to WM contrast (Ahmed et al., 2015), with lesions correctly identified in 6 out of 7 patients with positive and 14 out of 28 patients with negative MRI FCD reports. The average volume coverage reported was 
$20.14 \%$ for positive and $2.47 \%$ for negative MRI, and the false-positive rates were $2.4 \%$ and $1.04 \%$, respectively.

In comparison with our method, the texture feature approach is close to the morphologic approach regarding results, and with much simpler computational methods and less computational time required.

\section{Conclusion}

We developed a classifier capable of identifying type II DCF in axial FLAIR MRI images of a patient with this type of lesion. The proposed method obtained specificity 0.81 and sensitivity 0.87 in the tested individuals.

Comparisons with state-of-the-art methods show that there is still no clinical tool capable of satisfying the necessary conditions for extensive use. Existing studies have limitations ranging from non-standardized image acquisition protocols to dependence on automatic methods of image preprocessing without complete effectiveness.

\section{References}

Adler, S., Wagstyl, K., Gunny, R., Ronan, L., Carmichael, D., Cross, J. H., ... Baldeweg, T. (2017). Novel surface features for automated detection of focal cortical dysplasias in pediatric epilepsy. NeuroImage: Clinical, 14, 18-27. http://doi.org/10.1016/j.nicl.2016.12.030

Ahmed, B., Brodley, C. E., Blackmon, K. E., Kuzniecky, R., Barash, G., Carlson, C., ... Thesen, T. (2015). Cortical feature analysis and machine learning improves detection of MRI-negative focal cortical dysplasia. Epilepsy \& Behavior, 48, 21-28. http://doi.org/10.1016/j.yebeh.2015.04.055

Antel, S. B., Collins, D. L., Bernasconi, N., Andermann, F., Shinghal, R., Kearney, R. E., ... Bernasconi, A. (2003). Automated detection of focal cortical dysplasia lesions using computational models of their MRI characteristics and texture analysis. NeuroImage, 19(4), 1748-1759. http://doi.org/10.1016/S1053-8119(03)00226-X

Bergo, F., \& Falcao, A. (2008). FCD segmentation using texture asymmetry of MR-T1 images of the brain. 2008 5th IEEE International Symposium on Biomedical Imaging: From Nano to Macro, 424-427. Retrieved from http://ieeexplore.ieee.org/xpls/abs_all.jsp?arnumber=4541023

Bernasconi, A., Antel, S. B., Collins, D. L., Bernasconi, N., Olivier, A., Dubeau, F., ... Arnold, D. L. (2001). Texture analysis and morphological processing of magnetic resonance imaging assist detection of focal cortical dysplasia in extra-temporal partial epilepsy. Ann Neurol, 49(6), 770-775. http://doi.org/10.1002/ana.1013

Blümcke, I., Aronica, E., Miyata, H., Sarnat, \#harvey B, Thom, M., Roessler, K., ... Spreafico, R. (2016). International recommendation for a comprehensive neuropathologic workup of epilepsy surgery brain tissue: A consensus Task Force report from the ILAE Commission on Diagnostic Methods. Epilepsia, 57(3), 348358. http://doi.org/10.1111/epi.13319

Blümcke, I., Thom, M., \& Aronica, E. (2011). The clinicopathologic spectrum of focal cortical dysplasias: A consensus classification proposed by an ad hoc Task Force of the ILAE Diagnostic Methods. ..., 52(1), 158-174. http://doi.org/10.1111/j.1528- 


\subsubsection{7.x.The}

Cantor-Rivera, D., Khan, A. R., Goubran, M., Mirsattari, S. M., \& Peters, T. M. (2015). Detection of temporal lobe epilepsy using support vector machines in multiparametric quantitative MR imaging. Computerized Medical Imaging and Graphics, 41, 14-28. http://doi.org/10.1016/j.compmedimag.2014.07.002

Carass, A., Roy, S., Jog, A., Cuzzocreo, J. L., Magrath, E., Gherman, A., ... Pham, D. L. (2017). Longitudinal multiple sclerosis lesion segmentation: Resource and challenge. NeuroImage, 148, 77-102. http://doi.org/10.1016/j.neuroimage.2016.12.064

Colombo, N., Tassi, L., Galli, C., Citterio, A., Lo Russo, G., Scialfa, G., \& Spreafico, R. (2003). Focal cortical dysplasias: MR imaging, histopathologic, and clinical correlations in surgically treated patients with epilepsy. AJNR. American Journal of Neuroradiology, 24(4), 724-33. Retrieved from http://www.ncbi.nlm.nih.gov/pubmed/12695213

Duncan, J. S., Winston, G. P., Koepp, M. J., Ourselin, S., John, P., Frcp, S. D., ... Ourselin, P. S. (2016). Brain imaging in the assessment for epilepsy surgery. The Lancet Neurology, 15(4), 420-433. http://doi.org/10.1016/S1474-4422(15)00383-X

Fauser, S., Schulze-Bonhage, A., Honegger, J., Carmona, H., Huppertz, H.-J., Pantazis, G., ... Zentner, J. (2004). Focal cortical dysplasias: surgical outcome in 67 patients in relation to histological subtypes and dual pathology. Brain: A Journal of Neurology, 127(Pt 11), 2406-18. http://doi.org/10.1093/brain/awh277

Fisher, R. S., Acevedo, C., Arzimanoglou, A., Bogacz, A., Cross, J. H., Elger, C. E., ... Wiebe, S. (2014). A practical clinical definition of epilepsy. Epilepsia, 55(4), 475482. http://doi.org/10.1111/epi.12550

Fisher, R. S., Cross, J. H., French, J. A., Higurashi, N., Hirsch, E., Jansen, F. E., ... Zuberi, S. M. (2017). Operational classification of seizure types by the International League Against Epilepsy: Position Paper of the ILAE Commission for Classification and Terminology. Epilepsia, 58(4), 522-530. http://doi.org/10.1111/epi.13670

Francione, S., Vigliano, P., Tassi, L., Cardinale, F., Mai, R., Lo Russo, G., \& Munari, C. (2003). Surgery for drug resistant partial epilepsy in children with focal cortical dysplasia: anatomical-clinical correlations and neurophysiological data in 10 patients. Journal of Neurology, Neurosurgery, and Psychiatry, 74(11), 1493-501. http://doi.org/10.1136/jnnp.74.11.1493

Hatt, M., Laurent, B., Ouahabi, A., Fayad, H., Tan, S., Li, L., ... Visvikis, D. (2018). The first MICCAI challenge on PET tumor segmentation. Medical Image Analysis, 44, 177-195. http://doi.org/10.1016/j.media.2017.12.007

Hildebrandt, M., Pieper, T., Winkler, P., Kolodziejczyk, D., Holthausen, H., Blümcke, I., \& Blu, I. (2005). Neuropathological spectrum of cortical dysplasia in children with severe focal epilepsies. Acta Neuropathologica, 110(1), 1-11. http://doi.org/10.1007/s00401-005-1016-6

Hong, S. J., Kim, H., Schrader, D., Bernasconi, N., Bernhardt, B. C., \& Bernasconi, A. (2014). Automated detection of cortical dysplasia type II in MRI-negative epilepsy. Neurology, 83(1), 48-55. http://doi.org/10.1212/WNL.0000000000000543 
Hotelling, H. (1933). Analysis of a complex of statistical variables into principal components. Journal of Educational Psychology, 24(6), 417-441. http://doi.org/10.1037/h0071325

Huppertz, H.-J. (2013). Morphometric MRI Analysis. In Dysphagia (Vol. 39, pp. 7384). http://doi.org/10.1007/174_2012_564

Kini, L. G., Gee, J. C., \& Litt, B. (2016). Computational analysis in epilepsy neuroimaging: A survey of features and methods. NeuroImage: Clinical, 11(1), 515529. Statistical Mechanics; Mathematical Physics; Mathematical Physics. http://doi.org/10.1016/j.nicl.2016.02.013

Krsek, P., Maton, B., Korman, B., Pacheco-Jacome, E., Jayakar, P., Dunoyer, C., ... Duchowny, M. (2008). Different features of histopathological subtypes of pediatric focal cortical dysplasia. Annals of Neurology, 63(6), 758-69. http://doi.org/10.1002/ana.21398

Menze, B. H., Jakab, A., Bauer, S., Kalpathy-Cramer, J., Farahani, K., Kirby, J., ... Van Leemput, K. (2015). The Multimodal Brain Tumor Image Segmentation Benchmark (BRATS). IEEE Transactions on Medical Imaging, 34(10), 1993-2024. http://doi.org/10.1109/TMI.2014.2377694

Pearson, K. (1901). LIII. On lines and planes of closest fit to systems of points in space. The London, Edinburgh, and Dublin Philosophical Magazine and Journal of Science, 2(11), 559-572. http://doi.org/10.1080/14786440109462720

Ramli, N., Rahmat, K., Lim, K. S., \& Tan, C. T. (2015). Neuroimaging in refractory epilepsy. Current practice and evolving trends. European Journal of Radiology, 84(9), 1791-1800. http://doi.org/10.1016/j.ejrad.2015.03.024

Roy, H., Lippé, S., Lussier, F., Sauerwein, H. C., Lortie, A., Lacroix, J., \& Lassonde, M. (2011). Developmental outcome after a single episode of status epilepticus. Epilepsy \& Behavior: E\&B, 21(4), 430-6. http://doi.org/10.1016/j.yebeh.2011.05.009

Sheppard, E., \& Lippé, S. (2012). Cognitive outcome of status epilepticus in children. Epilepsy Research and Treatment, 2012, 984124. http://doi.org/10.1155/2012/984124 